\title{
Model-Based Opportunistic Channel Access in Dynamic Spectrum Access Networks
}

\author{
Manuj Sharma*†, Anirudha Sahoo* and K. D. Nayak ${ }^{\dagger}$ \\ *Indian Institute of Technology Bombay, Mumbai, India \\ Email: \{manuj,sahoo\}@it.iitb.ac.in \\ $\dagger$ Advanced Numerical Research and Analysis Group \\ DRDO, Hyderabad, India
}

\begin{abstract}
We propose a model-based channel access mechanism for cognitive radio-enabled secondary network, which opportunistically uses the channel of an unslotted primary network when the channel is sensed idle. We have considered IEEE 802.11 WLAN as a de facto primary network operating in ISM band. Our study focuses on a single WLAN channel that is used by WLAN clients and a WLAN server for a mix of Email, FTP, and HTTP-based web browsing applications. We model the occupancy of the channel by primary WLAN nodes as an alternating renewal process. The secondary node uses the model to estimate residual idle time duration after the channel is sensed idle, and opportunistically transmits frames in that duration. Our simulation results show that the performance of secondary network is sensitive to the channel sensing duration and that high secondary throughput can be achieved without affecting the primary network significantly by choosing appropriate value of channel sensing duration.
\end{abstract}

\section{INTRODUCTION}

Opportunistic Spectrum Access (OSA) has emerged as a promising approach to efficiently utilize the electromagnetic spectrum. In this approach, the secondary users use those parts of the spectrum band that are not currently utilized in space or time by any primary user. In this paper, we propose a modelbased approach for opportunistic access of unslotted primary network channel by secondary network nodes. Each secondary node collects the statistical data about the channel occupancy by primary nodes. It then fits an appropriate distribution to the statistical data to construct a channel occupancy model and proceeds with the algorithms presented in this paper to opportunistically use the channel. We refer to primary network as the network which carry the main traffic in a designated spectrum band. For our work, we have considered a 802.11based WLAN as a de facto primary network operating in ISM band. WLAN devices constitute the primary devices in this band, whereas other networks, such as Bluetooth and sensor networks, which operate in these bands, can be treated as secondary networks (see [1]). The secondary network considered in this paper is an unslotted network. We have constructed and applied the model for a single WLAN primary channel, but the approach can be used for multichannel primary networks as well. As a part of our future work, we will implement the algorithm for other multiple channel primary networks such as WiMAX, GSM or TV network.

\section{NOTATIONS}

In this section, we introduce the main notations used in rest of the paper.

$I, B, C$ : Random variables denoting channel idle period, busy period and cycle time respectively. $(C=I+B)$

$\xi(t)$ : Ending time of the last (previous) renewal cycle at sensing time $t$.

$p h_{I}, p h_{B}$ : Number of phases in Hyperexponential distribution (HED) for idle time random variable $I$ and busy time random variable $B$ respectively.

$\lambda_{i}, \alpha_{i}$ : Rate parameter and probability value respectively of the $i^{\text {th }}$ phase (exponential) of the HED for random variable $I .\left(i=1 \ldots p h_{I}\right.$, and $\left.\sum_{i=1}^{p h_{I}} \alpha_{i}=1\right)$

$\mu_{i}, \beta_{i}$ : Rate parameter and probability value respectively of the $i^{\text {th }}$ phase (exponential) of the HED for random variable $B .\left(i=1 \ldots p h_{B}\right.$, and $\left.\sum_{i=1}^{p h_{B}} \beta_{i}=1\right)$

$H E D\left(p h_{I}, \lambda_{i}, \alpha_{i}\right)$ : Denotes Hyperexponential distribution (for random variable $I$ ) with parameters $\left(p h_{I}, \lambda_{i}, \alpha_{i}\right)$, where $i=1 \ldots p h_{I}$.

$H E D\left(p h_{B}, \mu_{i}, \beta_{i}\right)$ : Denotes Hyperexponential distribution (for random variable $B$ ) with parameters $\left(p h_{B}, \mu_{i}, \beta_{i}\right)$, where $i=1 \ldots p h_{B}$.

$T_{o b s}$ : Denotes the duration for which secondary nodes passively sense the channel to gather occupancy statistics (idle and busy time durations) of the channel by primary nodes.

$S(t)$ : A boolean variable that denotes the outcome of sensing the channel at time instant $t$ by a secondary node. $S(t)$ can either take value $B U S Y$ or IDLE.

$T_{R I}(t)$ : A random variable that denotes residual idle time for the channel at sensing instant $t$.

$T h_{R I}$ : A predefined threshold for residual idle time; secondary node uses the channel opportunistically only if the estimated residual idle time is more than this threshold. $\left(T h_{R I}=\right.$ (one secondary frame transmission time + cushion time $) \approx 300$ microseconds $)$.

$T_{E R I}(t)$ : Effective residual idle time on the channel. This is computed as: $T_{E R I}(t)=$ $\min \left\{\left(T_{R I}(t)\right)_{\text {sender }},\left(T_{R I}(t)\right)_{r c v}\right\}$, where $\left(T_{R I}(t)\right)_{\text {sender }}$ is the residual channel idle time estimated by the sender at time $t$ and $\left(T_{R I}(t)\right)_{r c v}$ is the residual channel idle time at time instant $t$ as estimated by the receiver using 


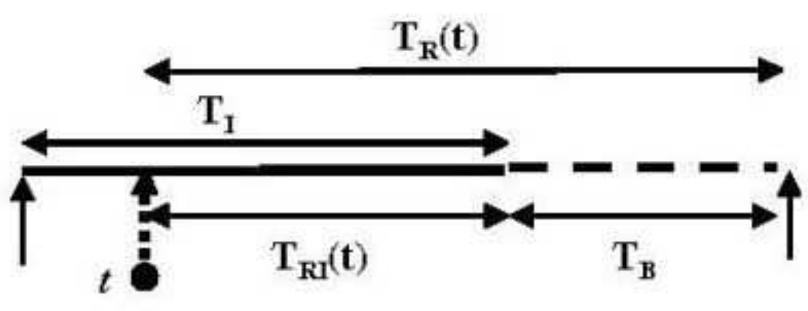

Fig. 1: Residual idle time at sensing instant $t$

TABLE I: Statistics of Channel Idle Time Samples and Fitted Distributions

\begin{tabular}{|l|c|c|c|}
\hline & Mean & Variance & CoV \\
\hline Collected Data Sample & 2.04179 & 132.95675 & 31.89235 \\
\hline EXPO & 2.04179 & 4.16892 & 1.00000 \\
\hline HED-2 & 2.03965 & 181.08033 & 43.52708 \\
\hline HED-4 & 2.03965 & 128.22349 & 30.82165 \\
\hline HED-5 & 2.03965 & 121.13900 & 29.11872 \\
\hline HED-10 & 2.03965 & 123.06167 & 29.58088 \\
\hline
\end{tabular}

channel occupancy model.

$S R T S$ : Secondary RTS frame

$S C T S$ : Secondary CTS frame

$C O N F$ : Confirmation frame

\section{Primary Network Channel Occupancy Model}

We treat a channel as a 2 -state system. We consider a channel to be idle (or available) (from SU's perspective) when it is not used by any primary user, and busy (or occupied) (from SU's perspective) when it is used by any primary user. Channel occupancy model is constructed by secondary user. In order to construct the channel occupancy model, each secondary node passively gathers the primary user's channel occupancy statistics (idle and busy durations) for $T_{\text {obs }}$ duration. Channel occupancy is modeled as an alternating renewal process (see [2]) in which a cycle, consisting of idle duration followed by busy duration, repeats (renews) in time. Renewal of a cycle is said to occur when the channel becomes idle (i.e. the primary user stops transmitting on the channel). A related concept of interest to our work is residual idle time. Consider Figure 1 in which $T_{I}$ (solid segment) and $T_{B}$ (dashed segment) denotes the idle time and busy time respectively within a cycle. $t$ denotes the instant at which the secondary node senses the channel. $T_{R}(t)$ denote the residual time of the complete cycle at instant $t$. If the channel is idle at instant $t$, then $T_{R I}(t)$ represents the residual idle time as observed at time $t$.

After gathering the primary network channel occupancy data during $T_{o b s}$ duration, each secondary node fits an appropriate distribution to idle and busy period data samples. Table I shows the mean, variance and coefficient of variation of idle time data samples, as well as exponential (EXPO) and Hyperexponential distributions (HED- $n$ ) fitted to these samples ( $n$ denotes the number of phases in the fitted HED). Statistics for busy time samples are not presented here due to space limitation, but interested readers can refer to [3] for details. Since the coefficient of variation of idle time samples is significantly

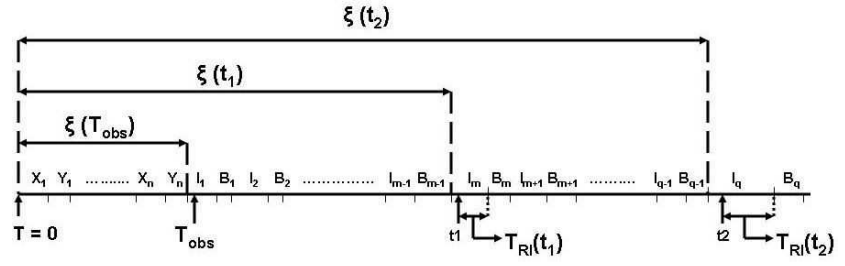

Fig. 2: Residual idle time computation

greater than 1, Hyperexponential distribution is a better fit for channel idle time. On the other hand, the variability $(\mathrm{CoV})$ of busy time samples is less than one (refer to [3]), so both HED and exponential distributions can provide a satisfactory fit for these samples. We have fitted 5-phase HEDs for both idle and busy time data samples in our simulations using Expectation Maximization (EM) algorithm proposed in [4]. Please refer to [3] for the parameter values of HED-5 distributions fitted to idle and busy time data.

\section{Model Based Channel Access Protocol}

In this section, we describe our proposed model-based channel access protocol.

\section{A. Residual Idle Time Computation Based on the Fitted Model}

Let us assume that during the channel observation period ( $\left.T_{\text {obs }}\right)$, a secondary node obtains the following idle and busy time data values: $X_{1}, Y_{1}, \ldots X_{n}, Y_{n}$, where $X_{i}$ represents the $i^{\text {th }}$ idle time data value and $Y_{i}$ represents the $i^{\text {th }}$ busy time data value for the channel (see Figure 2). Then the $i^{\text {th }}$ observed cycle time can be computed as : $c_{i}=X_{i}+Y_{i}$. If we use $\xi(t)$ to denote, in general, the ending time of the last renewal cycle prior to time $t$, then we can write $\xi\left(T_{\text {obs }}\right)=\sum_{j=1}^{n}\left(X_{j}+Y_{j}\right)=\sum_{j=1}^{n} c_{j}$. Note that $c_{n}$ is the last renewal cycle prior to $T_{o b s}$. If the first channel sensing by the secondary node occurs at time $t_{1}$ (where $t_{1}>T_{\text {obs }}$ ) and the channel is sensed idle, then the residual time at $t_{1}$ is computed as follows: Starting from the last renewal cycle prior to the previous observation/sensing time, (in this case, cycle $c_{n}$ prior to $T_{o b s}$ ), secondary node alternately generates idle and busy time random variable values using their respective distributions $\left(I \sim H E D\left(p h_{I}, \lambda_{i}, \alpha_{i}\right)\right.$ and $\left.B \sim H E D\left(p h_{B}, \mu_{i}, \beta_{i}\right)\right)$, till it generates $m^{\text {th }}$ idle time random variable value $\left(I_{m}\right)$, such that the current sensing time $t_{1}$ falls within the $m^{\text {th }}$ idle time (as shown in Figure 2). If we denote the $i^{\text {th }}$ generated cycle by $C_{i}$ (i.e. $C_{i}=I_{i}+B_{i}$ ), then $C_{m-1}$ is the last cycle prior to the current sensing time $t_{1}$, and $\xi\left(t_{1}\right)$ (the ending time of the last cycle $\left(C_{m-1}\right)$ prior to $\left.t_{1}\right)$ is calculated as: $\xi\left(t_{1}\right)=\xi\left(T_{o b s}\right)+\sum_{j=1}^{m-1}\left(I_{j}+B_{j}\right)=\xi\left(T_{o b s}\right)+\sum_{j=1}^{m-1} C_{j}$. From Figure 2, we see that the residual idle time at $t_{1}$ can be obtained as: $T_{R I}\left(t_{1}\right)=\left(\xi\left(t_{1}\right)+I_{m}\right)-t_{1}$. Extending the same explanation further, if the next sensing occurs at time $t_{2}$ and the channel is sensed idle, then the residual idle time at $t_{2}$ is computed as: $T_{R I}\left(t_{2}\right)=\left(\xi\left(t_{2}\right)+I_{q}\right)-t_{2}$, where $\xi\left(t_{2}\right)=\xi\left(t_{1}\right)+\sum_{j=m}^{q-1}\left(I_{j}+B_{j}\right)=\xi\left(t_{1}\right)+\sum_{j=m}^{q-1} C_{j}$, and $I_{q}$ is the $q^{\text {th }}$ idle time random variable value generated using modeled distributions such that the current sensing time $t_{2}$ falls within it (as shown in Figure 2). 

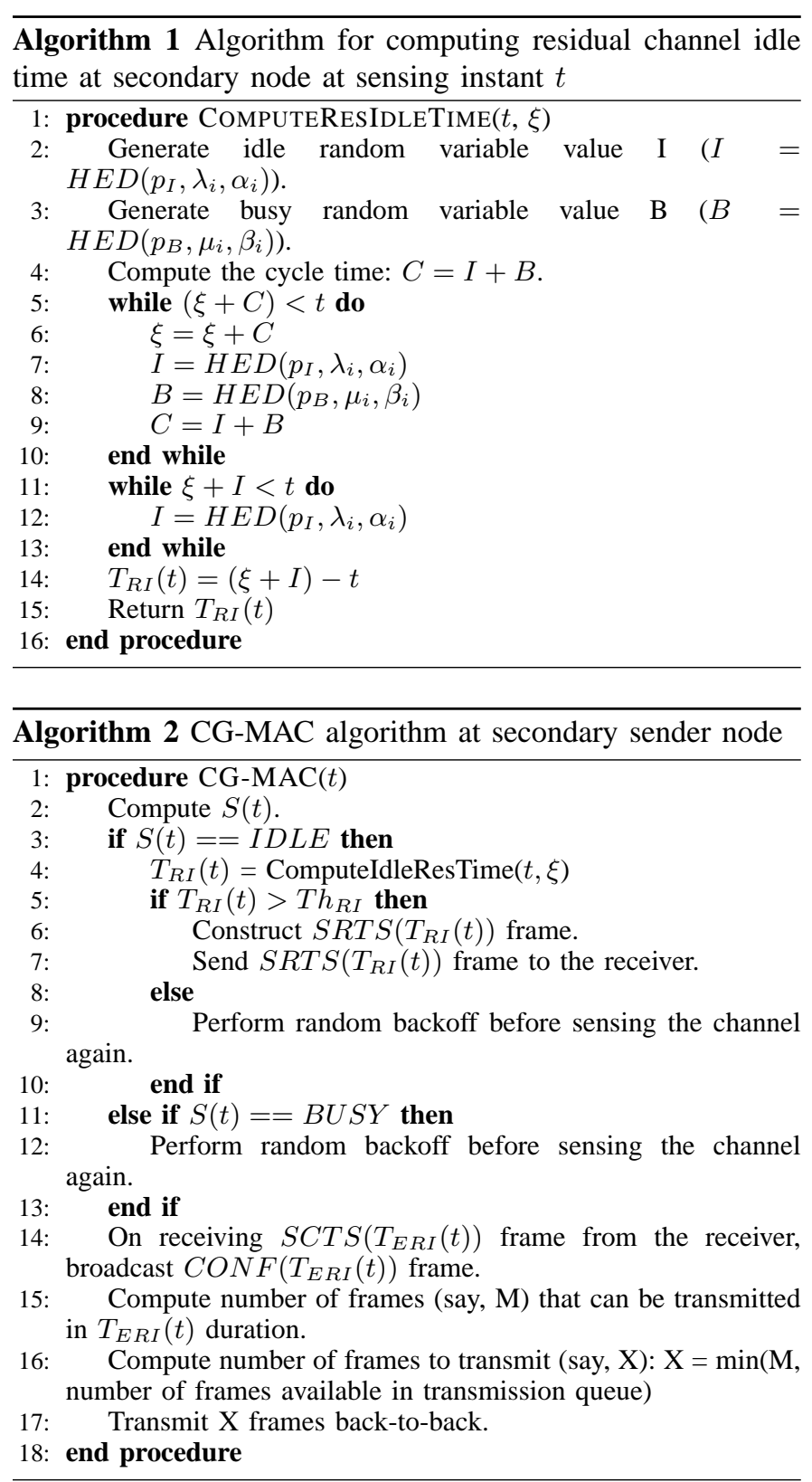

Algorithm for computing residual idle time by a secondary node at sensing instant $t$ and using the fitted channel idle and busy time models is given in Algorithm 1.

\section{B. The Protocol}

We assume that each secondary node has constructed channel occupancy model, as described in Section III. Whenever a secondary sender node has one or more frames to transmit at time instant $t$, its model-based channel access protocol senses the channel (line 2 of Algorithm 2), and if the channel is sensed idle, it estimates the residual idle time $\left(T_{R I}(t)\right)$ for the channel using the channel occupancy model (line 4). If the estimated residual idle time is more than a predefined threshold $T h_{R I}$ (line 5), it sends a SRTS frame (containing the estimated residual channel idle time) to the intended secondary receiver node (lines 6-7); otherwise it performs random backoff.

The receiver, on receiving the SRTS frame, senses the

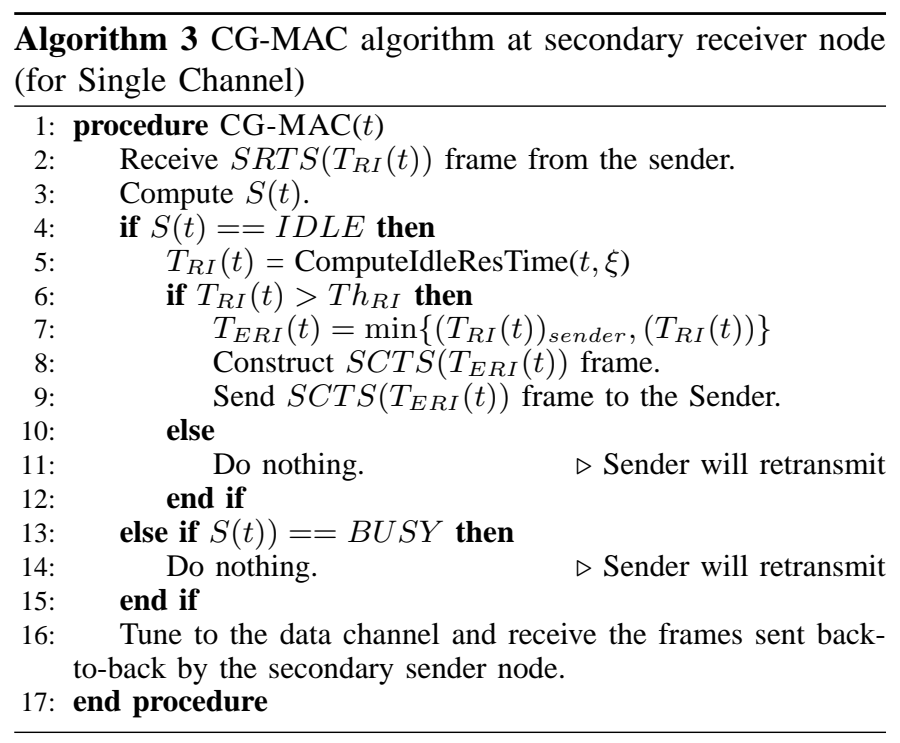

channel (line 3 of Algorithm 3), and if the channel is sensed idle (line 4), sends the estimated effective residual idle time back to the sender in an SCTS frame (lines 7-9). On receiving the SCTS frame, the secondary sender node broadcasts a CONF frame with the received Effective Residual Idle Time value (line 14 of Algorithm 2), and transmits the minimum of the number of the frames available in its transmission queue and the maximum number of frames that can be transmitted in the Effective Residual Idle Time duration (lines 15-17 of Algorithm 2). SRTS, SCTS and CONF frames are transmitted on a control channel.

\section{Simulation Results}

In this section, we discuss our simulation experiments and the results.

\section{A. Simulation Model}

We consider a 802.11 WLAN with a single data channel as a primary network. The channel is used by two primary WLAN client nodes (WLAN-Client-1 and WLAN-Client-2), and a primary WLAN server node (WLAN-Server). The WLANClient-1 node runs parallel sessions of FTP and Email applications. WLAN-Client-2 node runs a web browsing application. The WLAN-Server acts as a server for all the three types of applications. In addition to the primary WLAN, we consider one pair of secondary devices (a secondary sender node and a corresponding secondary receiver node) that opportunistically uses the channel. All the five nodes are within the transmission range of each other.

We use OPNET simulator [5] to simulate the model. We have used High load Email, High load FTP, and Heavy browsing HTTP application configurations provided by the simulator to run on the WLAN nodes. The send and receive interarrival times for emails are exponentially distributed with mean 360 seconds, whereas the email size is 2000 bytes (constant). Time between two file transfer requests is also exponentially distributed with mean 360 seconds, and the file size is 50000 bytes (constant). The percentage of file "get" commands to the total FTP commands is $50 \%$. For HTTP application, the time 
TABLE II: Main Simulation Parameters

\begin{tabular}{|l|c|}
\hline Parameters & Value \\
\hline Number of WLAN nodes & 3 \\
\hline Number of secondary nodes (SN) & 2 \\
\hline Simulation duration (Hours) & 8 \\
\hline $\begin{array}{l}T_{\text {obs }} \text { : Channel occupancy data } \\
\text { collection duration (by SN) }\end{array}$ & $\begin{array}{c}\text { First } \\
\text { WLAN channel number used in } \\
\text { simulation }\end{array}$ \\
\hline Channel data rate & 1 \\
\hline Channel bandwidth & $11 \mathrm{Mbps}$ \\
\hline Secondary node frame size (Bytes) & $22 \mathrm{MHz}$ \\
\hline $\begin{array}{l}\text { Clear Channel Assessment duration } \\
\text { for SN }\end{array}$ & 256 \\
\hline $\begin{array}{l}\text { Idle channel observation duration for SN } \\
\text { (COD) }\end{array}$ & $1.825 \mathrm{~ms}$ \\
\hline
\end{tabular}

between page requests is exponentially distributed with mean 60 seconds. Each page has 5 medium image objects and 1 constant object of 1000 bytes. The simulation runs for 8 hours. For the first 5 hours ( $T_{\text {obs }}$ duration), a sensor module within each secondary node passively senses the channel to gather occupancy statistics. This observed data consist of alternating sequence of channel idle and busy durations (due to WLAN applications transmissions). The secondary nodes construct primary network's channel occupancy model based on the observed occupancy data. During the model construction phase, the secondary node subsumes very small idle periods into the busy periods as these very small idle periods are too small to be useful for opportunistic transmissions by secondary nodes. In our simulations, the idle time values which are less than 75 microseconds are subsumed into busy time values. This threshold value is approximatlely equal to the transmission time of a very small WLAN frame, which is dominated mainly by DIFS time ( 50 microsec) and slot time ( 20 microsec).

Once the model is constructed, for next remaining 3 hours, the secondary node uses the channel model to estimate the residual channel idle durations and use the channel opportunistically along with the primary WLAN applications. A packet generator module within each secondary node generates Poisson traffic, with inter-frame arrival rate of 5000 frames/sec. Table II lists the main simulation parameters. We must emphasize that we have used a fixed observation window (of 5 hours) to construct the model in order to focus our investigation on performance of the model-based approach. In actual deployment of the algorithm, the model should be appropriately revised based on the data collected using new, possibly sliding, observation windows, as the traffic on the channel will change with time.

With the above simulation model, three different scenarios are simulated. The scenarios are explained below.

1) Scn-1 (Only P $\left.N_{W L A N}\right)$ : In this scenario, the secondary nodes are disabled and they do not transmit any traffic during the complete simulation. Only primary WLAN nodes running E-mail, FTP, and web browsing applications are operational. This scenario is the base case which provides us the performance of primary network in absence of any secondary network. Performance of primary network in this scenario can be compared with its performance in the other two scenarios (Scn-2 and Scn3 ) to assess the impact of opportunistic usage of WLAN channel by a secondary network.

2) Scn-2 $\left(P N_{W L A N}+S N_{W L A N}\right)$ : In this scenario, the secondary nodes uses WLAN protocol (i.e. secondary nodes are essentially WLAN nodes only) and do not construct any channel occupancy model. Instead, secondary sender node uses conventional WLAN protocol to exchange frames. In order to compare this scenario with other scenarios, the secondary network remain dormant for first 5 hours of simulation and becomes operational during last 3 hours of the simulation. This scenario enable us to compare the performance of secondary network when it shares the same network as a peer to the primary network.

3) Scn-3 $\left(P N_{W L A N}+S N_{M O D}\right)$ : In this scenario, the secondary nodes observe the channel for first 5 hours, and construct the channel occupancy model based on the gathered occupancy data. The secondary nodes fit 5-phase Hyperexponential distributions to the busy and idle periods, and construct Alternating Renewal Processbased channel occupancy model. Whenever the secondary sender node has one or more frames to transmit, and it senses the channel idle, it continues to sense the channel for a predefined channel observation duration. If the channel remains idle even for this additional duration, the node uses the channel occupancy model to predict the residual idle time on the channel (as described in Section IV-A) and transmits as many frames as possible in the predicted residual idle time.

An important parameter in the proposed model-based approach (Scn-3) is channel observation duration (COD). We assume that the idle periods that are greater than or equal to 1.0 seconds corresponds to durations when no application session is active. In between these large idle periods, there exist a sequence (called a block), of small idle and busy time values appearing alternatingly (which possibly corresponds to idle and busy times due to frame transmissions during active application sessions). The observed data file consist of several such blocks. The COD parameter should be set to such a value that enables the secondary node to exploit large idle times (when no application session is active) and avoid using small idle times that occur during frame transmissions of active application sessions, without decreasing secondary network throughput as much as possible. We study the impact of this parameter on the performance of secondary network. We set the value of COD parameter to four different values: 0 , value- 1 , value- 2 and value -3 . value -1 is obtained by taking the average of all the small idle time values $(<1.0$ seconds) that appear in the observed data file; value-2 is obtained by computing the average of small idle times $(<1.0$ seconds) in each block and taking the maximum of average values across all the blocks; value-3 is equal to the largest of the small 
idle time values $(<1.0)$ that appear in the observed data file. Setting COD $=0$ implies that once the secondary node senses the channel idle, it does not additionally senses the channel any longer. The performance of model-based approach for these four COD parameter values is given in the Result subsection.

\section{B. Performance Metrics}

We use the following performance metrics to assess the performance of model-based scenario $(S c n-3)$ and compare it with the other two scenarios (Scn-1 and Scn-2).

1) Average throughput of secondary network (frames/sec): This metric represents the average throughput of secondary network, which is achieved as a result of opportunistic transmission by secondary nodes. It is calculated only for scenarios $S c n-2$, and Scn-3. The metric computation is based on last 3 hours of simulation time during which the secondary network opportunistically uses the channel. In the first scenario $(S c n-1)$, the secondary nodes are not operational throughout the simulation, and therefore, the secondary network throughput is zero.

2) Average download response time for the e-mail application running on WLAN-Client-1 and WLAN-Server nodes

3) Average download response time for FTP application running on WLAN-Client-1 and WLAN-Server nodes

4) Average page response time of HTTP-based web browsing application running on WLAN-Client-2 and WLANServer nodes

The last three metrics show the impact of opportunistic transmissions by secondary nodes on the performance of primary WLAN applications.

\section{Results}

Main goal of the work reported in this paper is to study the throughput gains achieved by secondary network nodes by opportunistically transmitting on the primary WLAN channel, and to investigate the cost associated with such transmissions (in terms of user perceived impact on performance of the primary WLAN applications). Figure 3 shows the impact on primary HTTP application when model based approach is used in Scn-3 with different values of COD parameters (indicated within parenthesis). Similar impact is observed on primary Email and FTP applications also; for details please refer to [3]. Figure 4 shows the secondary network throughput obtained for these COD values in Scn-3. We note from these two figures that for COD values 0 and value-1, the achieved secondary network throughput is relatively high (Figure 4) but their impact on primary applications performance is extremely adverse (Figure 3). With COD parameter set to value-2 and value-3, the impact on primary applications is minimal, but the achieved secondary network throughput is less than that of value 0 and value- 1 case. However, the decrease in secondary throughput (with respect to COD value 0 or value-1) for the case with COD of value-2 is much smaller compared to that of value-3. Hence COD $=$ value -2 is a favorable COD value in terms of striking a balance between impact on primary network and drop in throughput of secondary network. In rest of our simulations for $S c n-3$, we use COD $=$ value-2. Figure 5 shows the secondary network throughput obtained in Scn-2 and Scn-3 (for secondary Poisson traffic rate of 5000 frames/sec). In Figure 6, Figure 7 and Figure 8, we show the impact on primary applications performance in scenarios $\mathrm{Scn}$ 1, Scn-2 and Scn-3 (with COD = value-2), when the secondary Poisson traffic rate (in $S c n-2$ and Scn-3) is 5000 frames/sec. These graphs show the time-averaged response time of primary applications. We note from these figures that response time of applications increase marginally when secondary nodes use model-based channel access (Scn-3) during last 3 hours of simulation time, as compared to the case when secondary nodes use WLAN protocol (Scn-2). On the other hand, the secondary network throughput obtained in $S c n-3$ is significantly larger (approximately 5 times) than secondary network throughput obtained in Scn-2 (Figure 5). The marginal increase in impact on primary applications performance in Scn-3 is too small to have noticeable effect on the application performance as perceived by the primary end user. This marginal increase is not very unexpected as the model-based scheme in Scn-3 uses stochastic channel model and is likely to make some impact on primary network performance because of its model-based probabilistic estimations. For primary networks, some amount of impact on the performance can be tolerated, especially when significant secondary network throughput (and consequently, higher channel utilization) can be achieved using model-based scheme. Please refer to [3] for results of lower Poisson traffic rates of secondary nodes, which do not have significant impact on primary.

\section{RELATED WORK}

A proactive spectrum access approach is proposed in [6] where secondary nodes take input from spectrum sensing modules, and build a three-tier predictive statistical models of spectrum availability on each channel. In [7], the authors have used renewal theory on past channel observations to estimate the probability that a channel will be idle in the next time slot. In [8], alternating renewal theory is used to analyse how often to sense the availability of licensed channel and in which order to sense those channels. In [9], authors address the issue of opportunistic spectrum access when multiple heterogeneous primary users are active simultaneously. Authors in [10] study the impact of various design options, such as sensing, packet length distribution and back-off time, on opportunistic spectrum access in cognitive radio networks. In [11], authors design strategies that decide, based only on knowledge of the channel bandwidths and data rates, which channels to probe in a multichannel wireless network for opportunistic transmission.

Coexistence of cognitive radio based devices and WLAN nodes has recently been studied in [12] using CTMC channel model and constrained MDP formulation. Opportunistic transmission (by slotted secondary network) in unslotted primary networks has also been recently studied in [13] and [14].

\section{CONCLUSION AND FUtURE WORK}

The results presented in this paper indicate that the modelbased approach to medium access has potential to deliver good 


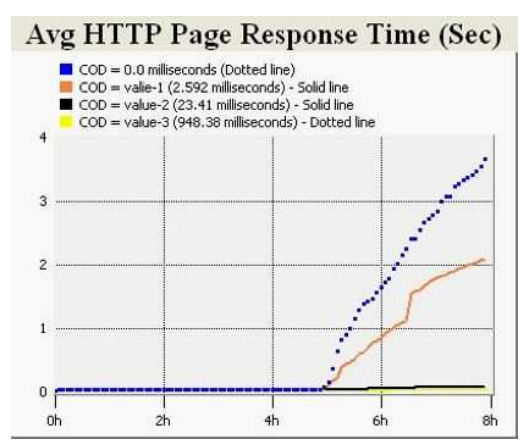

Fig. 3: HTTP Page Response Time (on Y-axis) in Scn-3 for Different COD values (Secondary Node (SN) Poisson Traffic Rate $=5000$ Frames/sec; X-axis: Simulation time in hours)

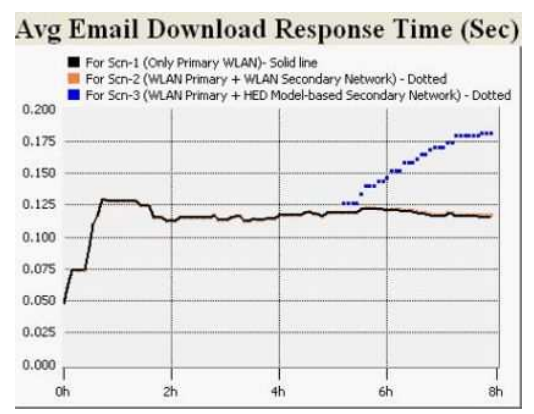

Fig. 6: Email Download Response Time (on Y-axis) in all Scenarios (SN Poisson Traffic Rate $=5000$ Frames $/ \mathrm{sec}$; X-axis: Simulation time in hours)

secondary network throughput without significantly affecting the performance of primary WLAN network. High secondary throughput can be achieved without affecting the primary network significantly by choosing appropriate value of channel sensing duration. The secondary network performance is better if the model is reasonably accurate.

In our future work, we plan to (i) investigate mechanism to guarantee upper bound on the impact on primary's performance, (ii) make the model online and adaptive based on changing channel occupancy statistics, (iii) extend and check the proposed algorithms for multiple pairs of secondary nodes, and (iv) develop a multi-channel opportunistic MAC protocol based on the channel access mechanism proposed in this paper. Additionally, we plan to study the impact of data outliers on model accuracy.

\section{REFERENCES}

[1] S. Geirhofer, L. Tong, and B. M. Sadler, "Dynamic Spectrum Access in the Time Domain: Modeling and Exploiting White Space," IEEE Communications Magazine, May 2007.

[2] K. S. Trivedi, Probability and Statistics with Reliability, Queuing and Computer Science Applications, 2nd ed. John Wiley and Sons, Inc., 2002.

[3] M. Sharma, A. Sahoo, and K. D. Nayak, "Model Based Opportunistic Transmission in Cognitive Radio Enabled Dynamic Spectrum Access Networks," Indian Institute of Technology (IIT) Bombay, Tech. Rep., March 2009. [Online].

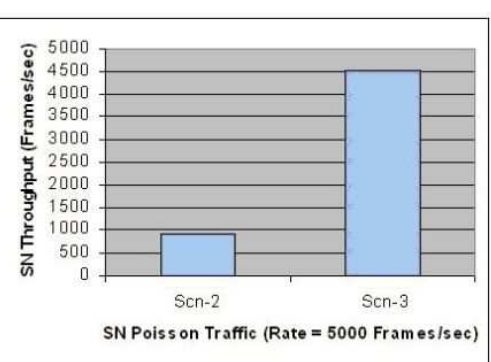

Fig. 5: Secondary Network Throughput (in Frames/Sec) for Scn-2 and Scn-3 Secondary Node (SN) Poisson Traffic Rate $=$ 5000 Frames/sec)

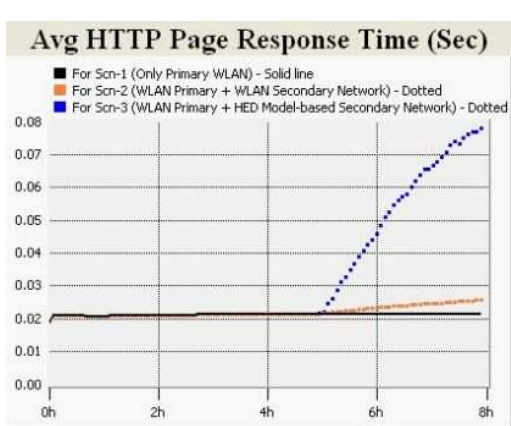

Fig. 8: HTTP Page Response Time (on Y-axis) in all Scenarios (Secondary Node Poisson Traffic Rate $=5000$ Frames/sec; $\mathrm{X}$-axis: Simulation time in hours)
Available: http://www.cse.iitb.ac.in/internal/techreports/reports/TR-CSE2009-22.pdf

[4] R. E. A. Khayari, R. Sadre, and B. R. Haverkort, "Fitting world-wide web request traces wth the EM-algorithm," Performance Evaluation (Elsevier), vol. 52, 2003.

[5] "Opnet network simulator," http://www.opnet.com.

[6] P. A. K. Acharya, S. Singh, and H. Zheng, "Reliable Open Spectrum Communications Through Proactive Spectrum Access," in IEEE TAPAS, Boston MA, August 2006.

[7] L. Yang, L. Cao, and H. Zheng, "Proactive channel access in dynamic spectrum networks," in Proceedings of CROWNCOM, 2007.

[8] H. Kim and K. G. Shin, "Efficient Discovery of Spectrum Opportunities with MAC-Layer Sensing in Cognitive Radio Networks," IEEE Transactions on Mobile Computing, vol. 7, no. 5, May 2008.

[9] E. Jung and X. Liu, "Opportunistic Spectrum Access in Heterogeneous User Environments," in Proceedings of 3rd IEEE Symposium on New Frontiers in Dynamic Spectrum Access Networks (IEEE DySPAN 2008), 2008.

[10] S. Huang, X. Liu, and Z. Ding, "Opportunistic Spectrum Access in Cognitive Radio Networks," in Proceedings of IEEE INFOCOM 2008, 2008.

[11] N. B. Chang and M. Liu, "Competitive Analysis of Opportunistic Spectrum Access Strategies," in Proceedings of IEEE INFOCOM 2008, 2008.

[12] S. Geirhofer, L. Tong, and B. M. Sadler, "Cognitive Medium Access: Constraining Interference Based on Experimental Models," IEEE Journal on Selected Areas in Communications, vol. 26, no. 1, January 2008.

[13] S. Shetty, M. Song, C. Xin, and E. K. Park, "A Learning-based Multiuser Opportunistic Spectrum Access Approach in Unslotted Primary Networks," in Proceedings of IEEE INFOCOM, 2009.

[14] Q. Zhao and K. Liu, "Detecting, Tracking, and Expoiting Spectrum Opportunities in Unslotted Primary Systems," in Proceedings of IEEE Radio and Wireless Symposium (RWS), 2008. 